Stability and efficiency of a CMOS sensor as detector of low energy $\beta$ and $\mathrm{y}$ particles

To cite this article: F. Collamati et al 2020 JINST 15 P11003

View the article online for updates and enhancements.

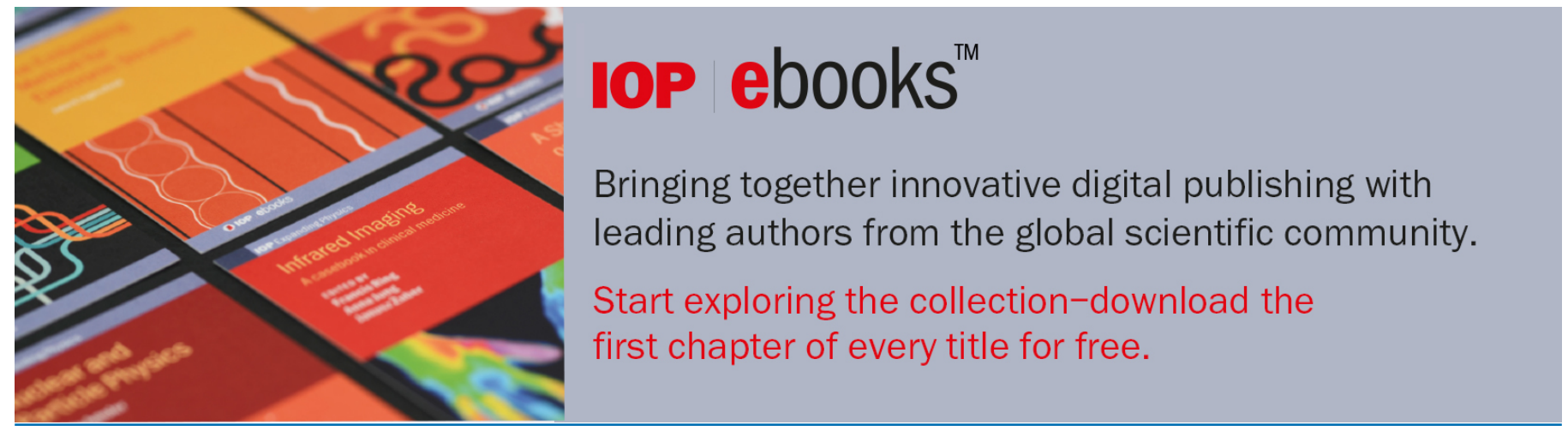




\title{
Stability and efficiency of a CMOS sensor as detector of low energy $\beta$ and $\gamma$ particles
}

\author{
F. Collamati, ${ }^{a}$ R. Amoruso, ${ }^{a, b, 1}$ L. Servoli, ${ }^{c}$ L. Alunni Solestizi, ${ }^{c, d}$ M. Biasini, ${ }^{c, d}$ V. Bocci, ${ }^{a}$ \\ C. Campeggi, ${ }^{c, d}$ P. De Maria, ${ }^{e}$ M. De Simoni, ${ }^{a, b}$ M. Fischetti, ${ }^{a, f}$ G. Franciosini, ${ }^{a, f}$ \\ K. Kanxheri, ${ }^{c}$ C. Mancini Terracciano, ${ }^{a, b}$ F. Meddi, ${ }^{a, b}$ M. Movileanu lonica, ${ }^{c}$ S. Morganti, ${ }^{a}$ \\ R. Mirabelli, ${ }^{a, b}$ E. Solfaroli Camillocci, ${ }^{a, e, 2}$ Á. Tóth ${ }^{g, h}$ and R. Faccini ${ }^{a, b}$ \\ a INFN Sezione di Roma, P.le Aldo Moro 2, Rome 00185, Italy \\ ${ }^{b}$ Dipartimento di Fisica, Sapienza University of Rome, \\ P.le Aldo Moro 5, Rome 00185, Italy \\ ${ }^{c}$ INFN Sezione Perugia, v. A. Pascoli, Perugia 06123, Italy \\ ${ }^{d}$ University of Perugia, Piazza Università 1, Perugia 06123, Italy \\ e Specialty School of Medical Physics, Sapienza University of Rome, \\ P.le Aldo Moro 5, Rome 00185, Italy \\ ${ }^{f}$ Dipartimento di Scienze di Base e Applicate per l'Ingegneria, Sapienza University of Rome, \\ P.le Aldo Moro 5, Rome 00185, Italy \\ ${ }^{\circ}$ Oncology Institute of Vojvodina, \\ Put doktora Goldmana 4, Sremska Kamenica 21204, Serbia \\ ${ }^{h}$ Institute of Nuclear Sciences Vinca, \\ Mike Petrovic Alasa 12-14, Belgrade 11351, Serbia \\ E-mail: elena.solfaroli@roma1.infn.it
}

${ }^{1}$ Co-first author.

${ }^{2}$ Corresponding author. 
AвstRACT: Radio Guided Surgery (RGS) is a nuclear medicine technique allowing the surgeon to identify tumor residuals in real time with a millimetric resolution, thanks to a radiopharmaceutical as tracer and a probe as detector. The use of $\beta^{-}$emitters, instead of $\gamma$ or $\beta^{+}$, has been recently proposed with the aim to increase the technique sensitivity and reducing both the administered activity to the patient and the medical exposure.

In this paper, the possibility to use the commercial CMOS Image Sensor MT9V115, originally designed for visible light imaging, as $\beta^{-}$radiation detector RGS is discussed.

Being crucial characteristics in a surgical environment, in particular its stability against time, operating temperature, integration time and gain has been studied on laboratory measurements.

Moreover, a full Monte Carlo simulation of the detector has been developed. Its validation against experimental data allowed us to obtain efficiency curves for both $\beta$ and $\gamma$ particles, and also to evaluate the effect of the covering heavy resin protective layer that is present in the "off the shelf" detector.

This study suggests that a dedicated CMOS Image Sensor (i.e. one produced without the covering protective layer) represents the ideal candidate detector for RGS, able to massively increase the amount of application cases and the efficacy of this technique.

KEYWORDS: Intra-operative probes; Image reconstruction in medical imaging 


\section{Contents}

1 Introduction 1

2 CMOS Image Sensors 2

3 Materials and methods $\quad 4$

3.1 Setup 4

3.2 Sources 4

3.3 Data analysis algorithm 5

$\begin{array}{ll}3.4 & \text { Monte Carlo simulation }\end{array}$

4 Measurements $\quad 7$

4.1 Stability 7

4.1.1 True Black level and gain $\quad 8$

4.1.2 Integration time 11

$\begin{array}{lll}4.1 .3 & \text { Temperature } & 12\end{array}$

$\begin{array}{lll}4.1 .4 & \text { Time } & 13\end{array}$

$\begin{array}{ll}\text { 4.2 Monte Carlo tuning } & 13\end{array}$

$\begin{array}{lll}4.3 & \text { Efficiency curves } & 15\end{array}$

$\begin{array}{lll}\text { 4.3.1 Without protective layer } & 16\end{array}$

$\begin{array}{ll}\text { 4.3.2 With protective layer } & 17\end{array}$

$\begin{array}{lll}4.4 \text { Conclusions } & 19\end{array}$

\section{Introduction}

Radio Guided Surgery (RGS) is a technique that aims at helping the surgeon to achieve a complete tumor resection. The technique is based on the administration to the patient, before surgery, of a radio pharmaceutical that bounds to tumoral cells and emits radiation. The detection of this emission by means of a dedicated detector, named probe, guides the surgeon towards tumor remnants.

In the last years, a novel approach to this technique has been proposed [1]. It exploits $\beta^{-}$ emitting radio tracers, aimimg at overtaking some limitations of the established technique, that relies on photon emitting isotopes ( $\gamma$-RGS) [2,3]. In fact, electrons with $\sim 1 \mathrm{MeV}$ energy traverse only few millimetres of human tissue, while about $1 / 3$ of $144 \mathrm{keV}$ photons (emitted by the commonly used ${ }^{99 m} \mathrm{Tc}$ ) penetrate more than $8 \mathrm{~cm}$, making $\gamma$-RGS difficult to apply in context characterised by elevated background coming from surrounding healthy tissue.

For such a purpose, an intraoperative detector for $\beta$ particles has been designed and developed, based on a high light yield and low density organic scintillator to achieve high efficiency to electrons while retaining low sensitivity to photons which, coming mainly from bremsstrahlung, are indeed a background in this context [1].

Extensive tests of this scintillating-detector have been performed on pure $\beta^{-}$emitters, like ${ }^{90} \mathrm{Sr}$, which emits a $0.546 \mathrm{MeV}$ electron decaying in ${ }^{90} \mathrm{Y}$, which in turn emits a $2.28 \mathrm{MeV}$ electron [4-7]. A feasibility study [8], based on Monte Carlo simulations, suggested the possibility to use also 
other isotopes like ${ }^{31} \mathrm{Si},{ }^{32} \mathrm{P},{ }^{97} \mathrm{Zr}$ and ${ }^{168} \mathrm{Re}$. All in all, very high efficiency to $\beta$ particles has been demonstrated, together with remarkable transparency to photons [9].

In particular, the first scintillator-based prototype of $\beta$ probe that has been used for the validation of the technique, demonstrated an efficiency to electrons reaching $70 \%$ at about $540 \mathrm{keV}$, being substantially blind to electrons below $\sim 300 \mathrm{keV}$ [8]. This threshold, however, is high enough to prevent the use of other possible pure $\beta^{-}$emitting isotopes, such as ${ }^{83} \mathrm{Br},{ }^{133} \mathrm{I}$ and ${ }^{153} \mathrm{Sm}$.

In this context, the development of a probe characterised by higher sensitivity to these low energy electrons would represent a crucial improvement to the technique, extending its application cases. To such a purpose, a viable approach is represented by the use as active element of the detector of a CMOS Image Sensor (CIS), which is expected to have very high sensitivity to low energy charged particles, while being almost transparent to low energy photons.

Indeed, this expected very low sensitivity to $\gamma$ particles suggest also the possibility to use this detector even with $\beta^{+}$emitting sources, where the contribution from annihilation photons becomes most significant.

This is in fact a potentiality that could exceptionally increase the number of application cases suitable for $\beta$-RGS. In fact, the main limitation to the efficacy of the proposed innovative technique is given by the lack of radio tracers that can be marked with pure $\beta^{-}$emitting isotopes, restricting the amount of tumors to which the technique can be applied.

On the contrary, being sensitive also to $\beta^{+}$emitters would open the way to the use of a pletora of radio tracers that are common in nuclear medicine for PET exams, such as ${ }^{18} \mathrm{~F}$ ( $\tau=110 \mathrm{~min}$, endpoint $0.633 \mathrm{MeV}$ ) or ${ }^{68} \mathrm{Ga}(\tau=68 \mathrm{~min}$, endpoint $1.9 \mathrm{MeV})$, and which already have numerous application cases.

Recent studies [10] demonstrate the interest in extending RGS application fields, but confirming that the traditional $\gamma$ approach is not suitable for high background contexts.

Moreover, it has been recently demonstrated $[11,12]$ by our team that ${ }^{68} \mathrm{Ga}-\mathrm{PSMA}$, an innovative radio pharmaceutical that is gaining more and more interest in the staging of prostate cancer, has for example an uptake good enough to perform $\beta$-RGS in lypmhoadenectomy in case of prostatic surgery.

Furthermore, it has to be stressed that if on one hand the proposed RGS technique leverages on the locality of the electron emission to gain sensitivity, on the other, it has in this very aspect also a possible limitation. As a matter of fact, the interposition of even small layers of normal tissue between the tumor and the probe could diminish particle energy, and eventually absorb it, possibly preventing the lesion to be detected.

In this context, a detector able to efficiently detect low energy $(\sim 100 \mathrm{keV}) \beta$ particles would be desirable both to use lower end point $\beta$ decaying nuclides and to detect deeper tumors.

In this work, a commercial-off-the-shelf CIS is characterised as a possible active element of such a detector. In particular, a quantification of its stability and efficiencies to both electrons and photons is presented.

\section{CMOS Image Sensors}

In the last decade, the CMOS (Complementary Metal-Oxide Semiconductor) technology has been growing at an impressive rate, to satisfy the consumer demand for more functionalities, including 
more advanced and robust imaging capabilities on Digital Still Cameras and smartphone cameras. Nowadays, several-milions-pixel devices are normally avaliable and the pixel size is of less than $2 \mu \mathrm{m}$. The sensitive layer below pixel surface has a thickness of some micrometers, similar to the size of the pixel. Beside imaging applications, new perspectives in various research areas could be opened by this class of devices: as an example, CMOS Image Sensors have already been tested as ionising radiation detectors for medical applications [13-15].

This type of sensors has an APS (Active Pixel Sensor) architecture, where each pixel hosts a photodiode and some transistors to implement the electronic front-end part. The functioning of each pixel is organized in a cycle subdivided into RESET Time, Integration Time, Readout Time. Each pixel photodiode is brought to a predefined voltage level during the RESET phase, then during the Integration Time it collects the electrons generated in the sensitive layer thus reducing its voltage. At the end of the Integration Time the pixel is readout, and the pixel signal for that integration time cycle is given by the voltage difference with respect to the voltage level at the end of the RESET. The output voltage of each pixel can be amplified by a built-in adjustable gain circuit and then converted by a 10 or 12-bit Analog-to-Digital Converter (ADC), hosted in the periphery of the matrix.

The output of the whole matrix constitutes a frame, as defined for visible imaging application. The average output of each pixel in dark condition is referred to as the pedestal for that pixel $\left(V_{i j}^{\text {ped }}\right)$, and is typically used to equalize the sensor response with respect to Fixed Pattern Noise. The single pixel output variance is the single pixel noise $\left(\sigma_{i j}\right)$, and is due to the fluctuations of the thermal e-h pair creation process in the sensitive volume of the pixel. This quantity is therefore a measurement of the intrinsic fluctuation of the dark response for that pixel, and will thus be used to discriminate the presence of a signal in the pixel: the higher is the single pixel noise, the smaller is the sensitivity for that pixel. The need to take pictures in poor visible light conditions leads to the reduction of this response fluctuation down to few electrons in the Integration Time interval.

Ionizing radiation is not sensitive to the difference among pixel color filters, because they are very thin and with similar density. Therefore the radiation passes through them and interacts with each pixel substrate in the same way. In this study, in order to equalize the response of the pixels, we set the sensor as a monochrome sensor and performed the acquisition in RAW mode. In this modality, the signal level of each pixel is read out separately and recorded without any manipulation by the sophisticated algorithms of the proprietary DAQ board.

An electron crossing the sensitive layer will deposit energy via the ionization mechanism, leading to the creation of many e-h pairs that will produce a sensible drop in the voltage of the collecting photodiode. For example a Minimum Ionising Particle (MIP) crossing the sensitive layer of the CIS produces few hundreds of e-h pairs [16]. The produced charge diffuses, and it is typically collected by just few pixels $(\sim 5)$ around the one with the maximum signal, forming a so called "cluster". Thanks to the low single pixel noise level, even the small amount of electrons collected by the photodiodes are enough to reach a S/N ratio of 20 and a detection efficiency of $\sim 100 \%$ [17].

In addition to this intrinsic very high sensitivity to charged particles, the small thickness of the sensitive layer reduces the photon interaction probability, especially for photon energies over $80 \mathrm{keV}$. For all these reasons, CIS can represent a very well-suited detector to discriminate between electrons and photons in a mixed radiation field.

In the recent past we started to investigate the possibility of using some MICRON (now Aptina Imaging) rolling shutter CIS as ionising radiation detector for charged particles. In this paper in 
particuar we report results concerning a standard VGA sensor, the MT9V115 [18], featuring a $648 \times 488$ pixel matrix, with a $1.75 \mu \mathrm{m}$ pixel size, and a few micrometers epitaxial layer depth. The integration time over which the signal is collected may vary up to $500 \mathrm{~ms}$. The gain of the electronic chain from single pixel to ADC input can be adjusted from 1.00 up to 31.5 and the analog-to-digital conversion system of 10 bits gives a dynamic range of 1024 ADC units. There is the possibility to vary the hardware offset (RGB True Black level $=42$ ) over the entire ADC range. For our purposes, needing monochromatic pixels, all the RGB gains must be set to the same value; the value 1 correspond to the largest possible dynamic range for each pixel, with a saturation level of about 40000 collected electrons. The sensor is hosted in a MT9SH06 evaluation board, whose readout is assured by an Aptina proprietary Demo2 board, connected via an USB cord to a PC to power the system, control the sensor and receive data.

Being originally thought as an image sensor, the CIS has been operated in a dark box to shield it from visible light. Moreover, an important point to mention is the presence of a protective $400 \mu \mathrm{m}$ transparent layer $\left(\rho=2.4 \mathrm{~g} / \mathrm{cm}^{3}\right)$ placed in front of the active area. Originally thought for shielding and stability purposes, this protective layer has a deep impact on the ability of this detector to reveal low energy particles, since for example electrons with energy lower than $\sim 400 \mathrm{keV}$ are not able at all to traverse it and thus do not reach the detector itself. While waiting to develop a technique to safely remove it, we performed the measurements with the protective layer and used Monte Carlo simulations to evaluate its impact on the efficiency. This evaluation is also very important to asses the relevance of the additional material of the envelope (typically plastic of some kind) that will be present in the probe prototype to isolate the sensor and its circuitry from the operating field.

\section{Materials and methods}

\subsection{Setup}

To perform the measurements described in this paper, a custom-made mechanical setup has been produced. As shown in figure 1, it allows two degrees of freedom for the relative movement between sensor and source. Once aligned with the center of the source, the sensor can be moved horizontally and vertically. The distance between the source and the sensor plane was kept constant at $2 \mathrm{~mm}$. During the acquisition, the setup is shielded from the visible light by means of a dark box. All the radiative sources were placed in a plastic holder, fixed to the mechanical setup.

A standard acquisition consisted of 3000 frames of $200 \mathrm{~ms}$, for a total of $600 \mathrm{~s}$ to allow the extraction of the sensor characteristics with small statistical errors.

\subsection{Sources}

The majority of the measurements in this study have been performed using a sealed ${ }^{90} \mathrm{Sr}$ flat source, distributed on a circular surface of $16 \mathrm{~mm}$ in diameter.

Moreover, $\gamma$ emitting sealed sources have also been used, in particular ${ }^{60} \mathrm{Co}$ and ${ }^{22} \mathrm{Na}$, housed at the center of a plastic disc of $25 \mathrm{~mm}$ diameter. Since their active area is of about $1 \mathrm{~mm}^{2}$, comparable with the active area of the CIS, when taking data with these sources several measurements were acquired rotating the plastic disc, in order to have an estimate of the error given by the uncertainty on the actual position of the active spot, found to be of $\sim 3 \%$. 


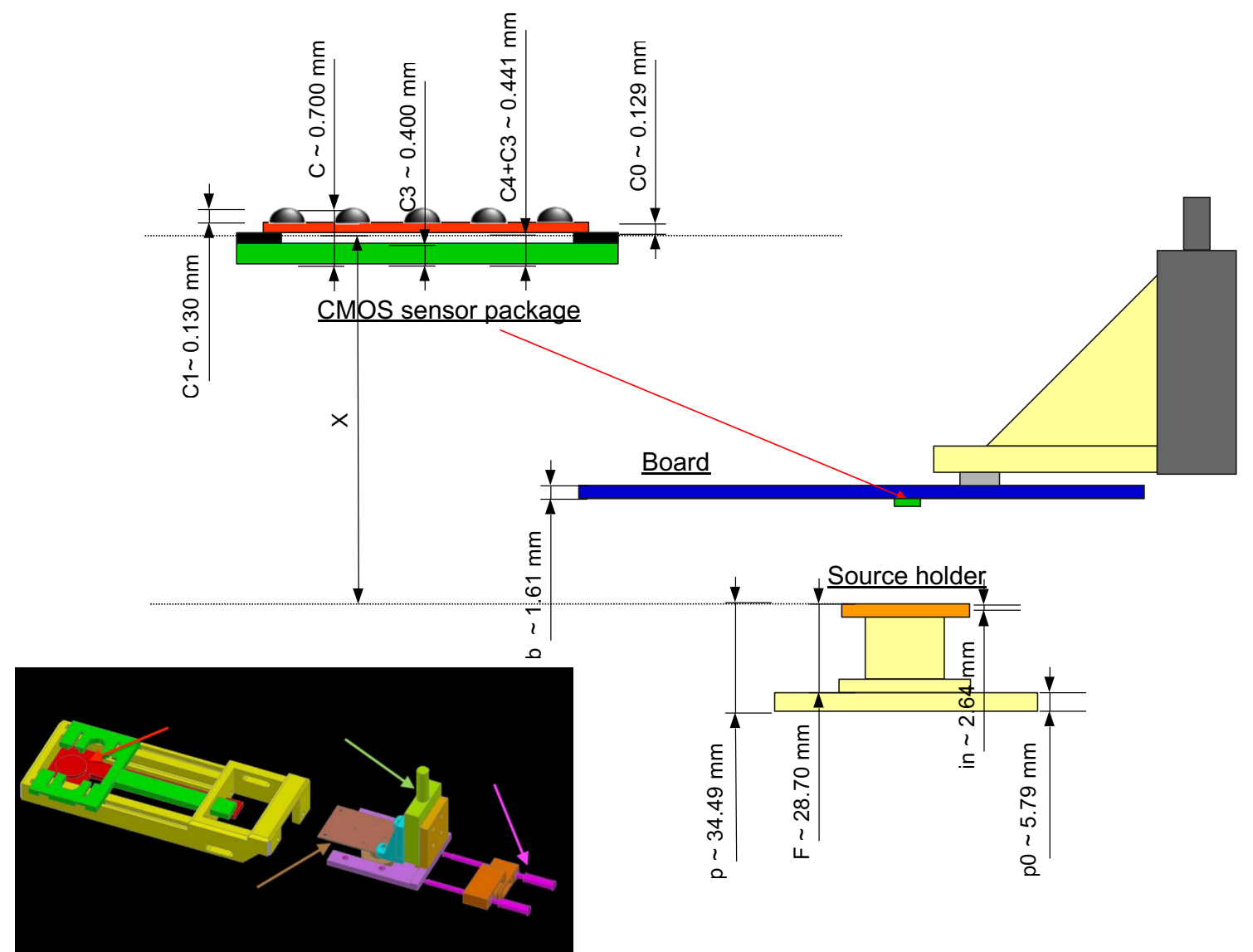

Figure 1. Experimental setup drawing: the structure allows two axis movements between the source and the detector, vertical $\mathrm{x}$-direction through the grey micrometric element at the far right, horizontal movement parallel to the PCB blue board through endless screw. The left bottom inset shows a picture of the setup with the source holder (red element and arrow) and the sensor board with sensor facing the source (brown element and arrow).

Lastly, also a source of ${ }^{90} \mathrm{Y}$ has been used, being this the most suited isotope for $\beta^{-}$-RGS [1]. In this case, however, constraints are put by the short decay time of this isotope $\left(\tau_{\mathrm{Y}}=92 \mathrm{~h}\right)$, that is not compatible with sealed sources. Therefore, liquid ${ }^{90} \mathrm{Y}$ from nuclear medicine treatments has been used to build cylindrical sources, adding to it agar agar, a polysaccharide employed as jellying agent. The resulting gel source has been placed in a dedicated plastic holder. By weighting this holder before and after the insertion of the material with a $1 \mathrm{mg}$ sensitive instrument, the net weight of the produced source has been obtained $(1.587 \pm 0.001 \mathrm{~g})$, and thus its volume, needed to assess the specific activity of the sample. To avoid the evaporation of the sample, that would change the volume and thus the geometry of the source, a plastic hermetic cover $8 \mu \mathrm{m}$ thick has been used. Characteristics of the sources are reported in table 1.

\subsection{Data analysis algorithm}

As already highlighted, the CIS used in this study is a commercial device intended to be used as image sensor. Therefore, its output is in form of "photos" or frames, i.e. $648 \times 488$ pixel matrices, 
Table 1. Table showing the characteristics of the utilised sources.

\begin{tabular}{|lccccccc|}
\hline & Nominal Activity & $\begin{array}{c}\text { Activity } \\
\text { at meas. } \\
(\mathrm{kBq})\end{array}$ & $\begin{array}{c}\text { Radius Active } \\
\text { Area } \\
(\mathrm{mm})\end{array}$ & Decay & $T_{1 / 2}$ & $P_{\text {emission }}$ & EP \\
& 2.54 & 2.3 & 8 & $\beta^{-}$ & $28 \mathrm{y}$ & $100 \%$ & 546 \\
${ }^{90} \mathrm{Sr}$ & 26.3 & 26.3 & 10.5 & $\beta^{-}$ & $64.24 \mathrm{~h}$ & $100 \%$ & 2280.1 \\
${ }^{90} \mathrm{Y}$ & 36.7 & 23.2 & 1 & $\beta^{-}$ & $5.27 \mathrm{~h}$ & $99.88 \%$ & 317.05 \\
${ }_{29}^{60} \mathrm{Co}$ & 40.5 & 16.0 & 1 & $\beta^{+}$ & $2.6 \mathrm{~h}$ & $90.3 \%$ & 545.67 \\
${ }_{21}^{22} \mathrm{Na}$ & & & & & & & \\
\hline
\end{tabular}

integrating the deposited energy in time windows of a given duration $\left(\Delta T_{\text {frame }}\right)$. However, in view of a possible application of this device as active element of a Radio Guided Surgery probe, which is basically a particle counter, a dedicated algorithm has been developed to transform this "photo-like" output into a "particle count" one.

The binary file produced by the data acquisition is converted into a Root file [19], which is then passed through a "reduction" algorithm in order to perform a zero-suppression of the data. Due to sources activity and detector size, in fact, of the more than $316^{\prime} 000$ pixels only very few are expected to have a particle signal in each frame. The reduction algorithm then defines the signal quantity for the $i-j$ pixel as:

$$
S_{i j}^{k}=\frac{V_{i j}^{k}-V_{i j}^{\mathrm{ped}}}{\sigma_{i j}},
$$

where $V_{i j}^{k}$ is the pixel value, $V_{i j}^{\text {ped }}$ is its pedestal and $\sigma_{i j}$ its noise, as defined previously in the text.

Figure 2a shows a submatrix of $30 \times 30$ pixel size with RAW data of each pixel after pedestal subtraction (i.e. just the numerator of the right side of eq. (3.1)), when the sensor is exposed to a ${ }^{90} \mathrm{Sr}$ source. Two peaks are visible, the left one with few pixels and the right one with just a single pixel, thus possibly suggesting the eventuality of a "two tracks signal". As a matter of fact, instead, the true $\beta^{-}$signature is the multipixel cluster, as shown in figure $2 \mathrm{~b}$ where the same frame is shown but with the variable $S_{i j}^{k}$ of eq. (3.1). The single pixel on the right of figure 2a has disappeared, because that pixel is characterised by a very large single pixel noise that allows very high fluctuations, while the multipixel structure on the left remained.

For this reason, only pixels with $S_{i j}^{k}$ above a given threshold are preserved and written in a "reduced" file. This file is then analyzed to find clusters by means of the procedure described in [20], giving finally a count of particles per frame interacting in the detector [21].

\subsection{Monte Carlo simulation}

In order to assist the design and interpretation of measurements described in this paper, a Monte Carlo simulation of the complete setup has been built in Geant4 [22].

In particular, each of the $316^{\prime} 224$ pixels is simulated, and also the mentioned covering layer is present, since its effect is one of the main topics to be addressed by the simulation. All the mentioned sources are also represented, both in terms of emitted particles, where the Geant 4 built-in database of radioactive isotopes has been used, and in terms of shape, material and size, which play a crucial 


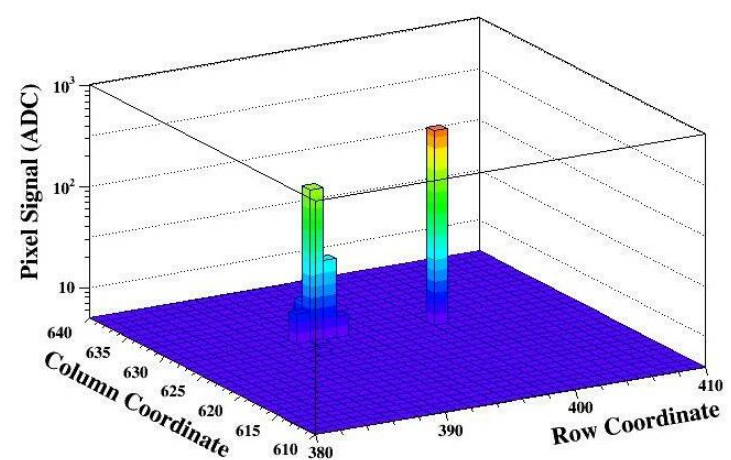

(a)

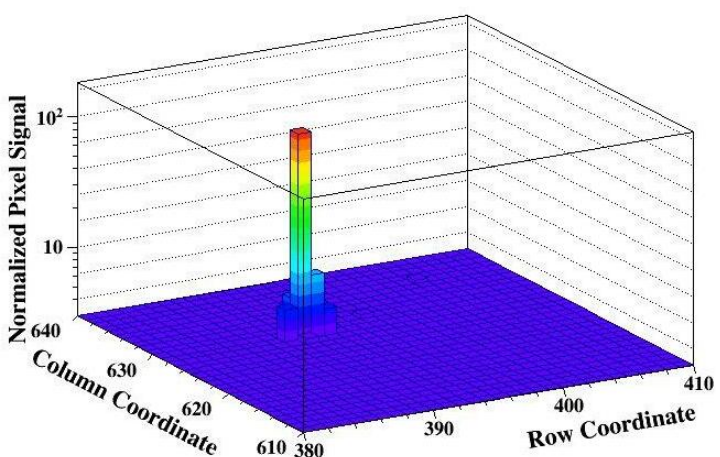

(b)

Figure 2. Event display for sensor exposed to a ${ }^{90} \mathrm{Sr}$ source: (a) a submatrix $30 \times 30$ pixel size where are reported the RAW data of each pixel after pedestal subtraction; (b) the same submatrix using quantity $S_{i j}^{k}$ of eq. (3.1).

role due to backscattering of emitted particles. For the same reason, also the PCB sensor board hosting the CMOS sensor has been inserted in the simulation.

G4RadioactiveDecayPhysics and G4EmLivermorePhysics packages were used for simulation of the physic processes. Due to the small pixel size and the high granularity needed, production cuts (i.e. the minimal range a particle has to have in order to be produced in the simulation) were manually lowered down to $0.01 \mu \mathrm{m}$ in the active volume and $10 \mu \mathrm{m}$ elsewhere. A random noise is also added to Monte Carlo results.

The simulation produces as output a .root file whose structure is the same of the data, allowing to send the Monte Carlo results through the same exact analysis algorithm of the data described above, gaining consistency and procedure simplification.

Within the simulation, there are basically two free parameters that have to be extracted from data. Firstly, the CMOS active area thickness $(\Delta Z)$, which is not known, but is expected to be of the order of few $\mu \mathrm{m}$. The second parameter is the conversion factor $(k)$ between the energy deposited in the detector (which is in $\mathrm{keV}$ in the MC) and the RAW data expressed in ADC counts.

To extract these parameters, a set of dedicated measurements have been performed, and will be discussed in this paper.

\section{Measurements}

\subsection{Stability}

Since the aim of this study is to evaluate the possibility to use a commercial CMOS sensor as active part of an intraoperative probe, it is crucial, when assessing its possible performances, to keep in mind the realistic application case scenario. Since a particularly important aspect is the stability of the detector's response, a dedicated series of measurements have been performed to study its behaviour as a function of temperature, time, gain and integration time. Starting values for the scans are those used in a previous work [20], i.e. nominal gain $=1$ (absolute gain $=1.31)$, integration time $=200 \mathrm{~ms}$, true black value of 42 . 


\subsubsection{True Black level and gain}

Just like every off-the-shelf camera, the CIS has a Gain $(G)$ level that can be set. This setting should not change the pedestal level and the contribution of the quantization error due to ADC conversion to the single pixel noise, while it will increase linearly both the signal and the dark current noise. Therefore, an elevated $G$ value implies a higher signal/noise ratio because signal will increase at a faster rate than the single pixel noise, at the price of a reduction of the dynamic range available before the saturation of the single pixel channel output. In a previous work [21] it has been found that the typical noise value at minimum gain for a CIS is of the order of $0.9 \mathrm{ADC}$, about half of which is not linked to the gain. Extrapolating this information to the maximum gain for MT9V115, 31.5 , we expect an average noise of 16-20 ADC at maximum gain. If we use the True Black default value of 42 , this could imply a deformation of the distributions given the distance from 0 of the order of $2 \sigma$.

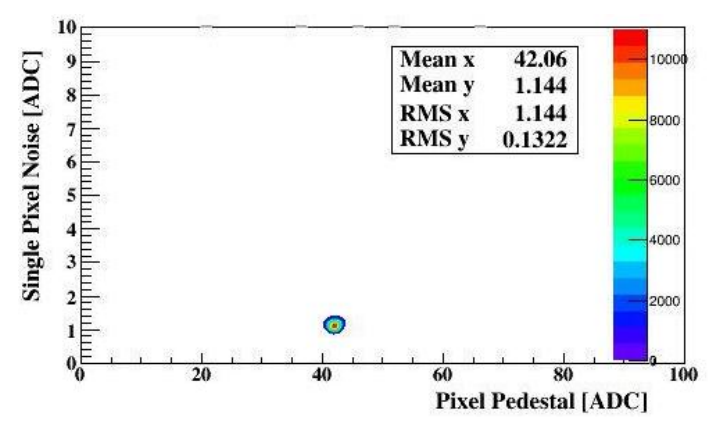

(a)

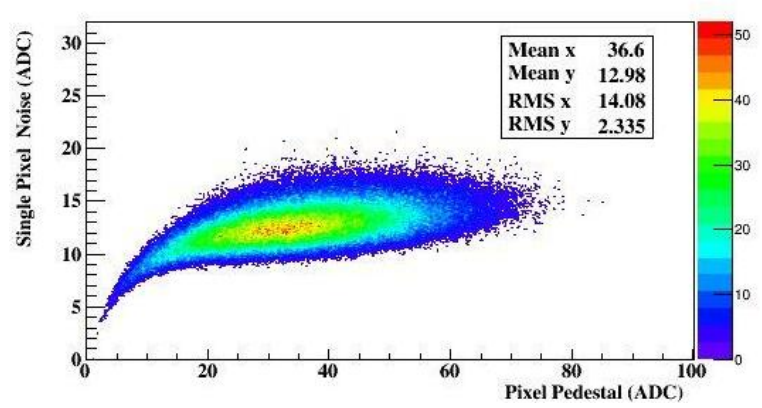

(b)

Figure 3. Correlation plot between pixel pedestal and noise at $200 \mathrm{~ms}$ integration time and a True Black level of 42: (a) gain $=1.31$; (b) gain $=31.5$. Note different vertical scales.

For example figure 3 shows for $200 \mathrm{~ms}$ integration time and True Black value of 42 the correlation plots between pixel pedestal and pixel noise respectively for minimum (1.31) and maximum (31.5) gain. The different behaviour at higher gain of the distribution shape is clear and points to the fact that some fluctuation of the pixel output value could indeed reach the lower limit of the dynamic range, distorting the computation of both the average pedestal and the average noise. In principle the change of the True Black level should not modify the noise and pedestal distributions, at least not in a significant way. A series of dedicated scans varying both True Black level from 37 to $84 \mathrm{ADC}$ and the gain from minimum (1.3) to maximum (31.5) has been done to verify this assumption and extract pedestal and noise values.

In table 2 are reported, with a $200 \mathrm{~ms}$ integration time, the average pixel pedestal $(\overline{\mathrm{Ped}})$ and the RMS of the distribution. We observe that, for a given gain, $\overline{\text { Ped }}$ is almost constant with respect to the corresponding True Black level, with the difference between the two slightly increasing with the gain. The last column of the table quotes the mean of the differences $\Delta=\overline{\mathrm{Ped}}-\mathrm{TB}$ for each TB level. Its RMS has been found to be smaller than $0.25 \mathrm{ADC}$. Also the width of the pedestal distribution is stable, confirming that True Black level in the scan range does not modify the distributions.

On the contrary, the gain setting does modify the average value of the pedestal distribution and also its RMS, as was shown in figure 3. Figure 4a shows the dependence of $\Delta$ on the gain, that 
Table 2. Mean and RMS of pixel pedestal distribution $(\overline{\mathrm{Ped}})$ varying True Black and pixel gain, with fixed integration time of $200 \mathrm{~ms}$. The last column shows the mean value of the difference $\Delta=(\overline{\mathrm{Ped}}-\mathrm{TB})$. All values are in ADC.

\begin{tabular}{|c|c|c|c|c|c|c|}
\hline \multirow{2}{*}{$\begin{array}{c}\text { Pixel } \\
\text { Gain }\end{array}$} & \multicolumn{5}{|c|}{$\overline{\mathrm{Ped}} \pm \mathrm{RMS}_{\mathrm{Ped}}$} & $\Delta$ \\
\hline 1.31 & $37.1 \pm 2.4$ & $42.1 \pm 2.5$ & $47.1 \pm 2.5$ & $63.1 \pm 2.6$ & $84.1 \pm 2.6$ & $0.1 \pm 0.2$ \\
\hline 2.63 & $37.2 \pm 4.6$ & $42.1 \pm 4.5$ & $47.1 \pm 4.3$ & $63.0 \pm 4.6$ & $84.0 \pm 4.7$ & $0.1 \pm 0.1$ \\
\hline 5.25 & $36.6 \pm 6.8$ & $41.8 \pm 7.0$ & $46.7 \pm 6.9$ & $62.8 \pm 6.9$ & $83.8 \pm 7.3$ & $-0.3 \pm 0.1$ \\
\hline 7.75 & $36.4 \pm 8.7$ & $41.3 \pm 8.3$ & $46.4 \pm 9.0$ & $62.4 \pm 8.6$ & $83.4 \pm 8.7$ & $-0.6 \pm 0.1$ \\
\hline 10.50 & $35.8 \pm 10.2$ & $40.7 \pm 10.1$ & $45.8 \pm 10.1$ & $61.9 \pm 10.2$ & $82.9 \pm 10.4$ & $-1.2 \pm 0.1$ \\
\hline 13.00 & $35.2 \pm 11.6$ & $40.3 \pm 11.9$ & $45.3 \pm 12.1$ & $61.1 \pm 11.7$ & $82.7 \pm 11.9$ & $-1.7 \pm 0.2$ \\
\hline 15.50 & $34.8 \pm 13.6$ & $39.9 \pm 13.5$ & $44.8 \pm 13.5$ & $60.9 \pm 13.4$ & $82.1 \pm 13.2$ & $-2.1 \pm 0.1$ \\
\hline 21.00 & $34.2 \pm 15.9$ & $38.9 \pm 16.1$ & $44.0 \pm 15.9$ & $59.5 \pm 15.9$ & $81.3 \pm 15.9$ & $-3.0 \pm 0.3$ \\
\hline 26.00 & $33.5 \pm 18.5$ & $38.0 \pm 18.6$ & $43.3 \pm 18.5$ & $59.6 \pm 19.0$ & $80.0 \pm 16.3$ & $-3.7 \pm 0.2$ \\
\hline 31.50 & $32.7 \pm 21.5$ & $37.5 \pm 21.5$ & $42.5 \pm 21.6$ & $58.2 \pm 21.9$ & $79.5 \pm 21.5$ & $-4.5 \pm 0.2$ \\
\hline
\end{tabular}

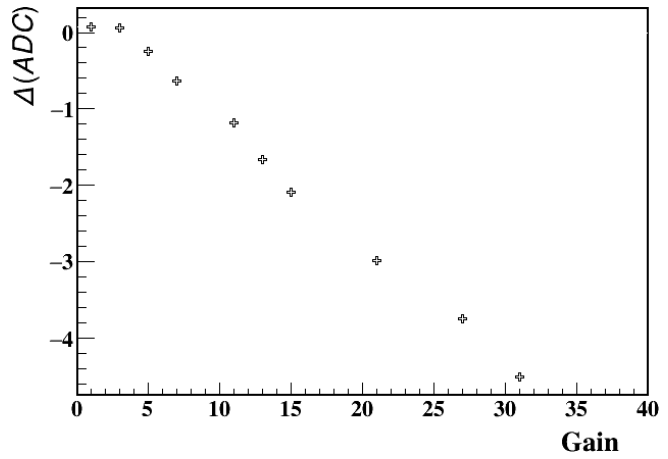

(a)

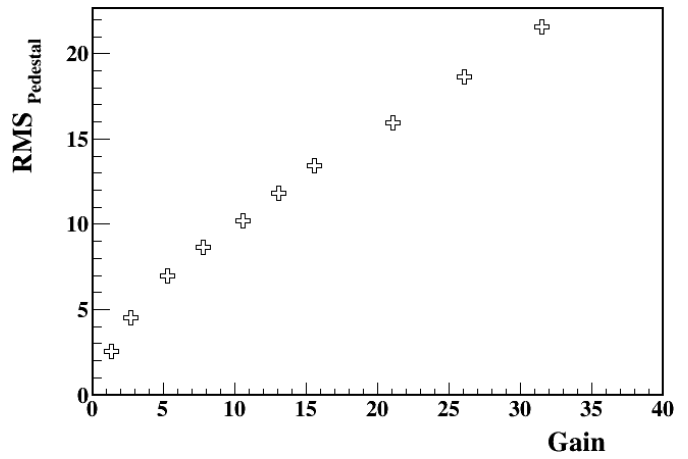

(b)

Figure 4. (a) Variation of average pixel pedestal vs. gain. (b) Variation of RMS of pedestal distribution vs. gain.

is essentially linear if we start from a gain greater than 3 , while it is constant for a gain below 3 . Therefore, to remain close to the expected neutral behaviour of the True Black level setting, the choice of the gain level should be less than 3. Another important aspect to evaluate is the widening of the pedestal dispersion as a function of the gain, shown in figure 4b: from few ADC at minimum gain it increases one order of magnitude for maximum gain, again independently from the True Black level choice (table 2). Since this wide dispersion is not desirable for the foreseen application, also from this point of view the choice of the working point for the gain should be restricted to the lowest values.

The same kind of study has been performed looking at the single pixel noise, and the results are reported in table 3. Also in this case the variation of True Black level has no consequences neither 
Table 3. Average single pixel noise $\left(\overline{\sigma_{\mathrm{SPN}}}\right)$ varying True Black level and pixel gain, with fixed integration time of $200 \mathrm{~ms}$. Last column reports the average of $\overline{\sigma_{\text {SPN }}}$ over all TB values and its RMS.

\begin{tabular}{|c|c|c|c|c|c|c|}
\hline Pixel & \multicolumn{5}{|c|}{ Average Single Pixel Noise $[\mathrm{ADC}]$} & $\bar{\sigma}$ \\
Gain & $\mathrm{TB}=37$ & $\mathrm{~TB}=42$ & $\mathrm{~TB}=47$ & $\mathrm{~TB}=63$ & $\mathrm{~TB}=84$ & {$[\mathrm{ADC}]$} \\
\hline 1.31 & $1.13 \pm 0.22$ & $1.15 \pm 0.15$ & $1.14 \pm 0.19$ & $1.15 \pm 0.18$ & $1.14 \pm 0.18$ & $1.14 \pm 0.01$ \\
\hline 2.63 & $1.71 \pm 0.31$ & $1.70 \pm 0.30$ & $1.74 \pm 0.30$ & $1.72 \pm 0.32$ & $1.71 \pm 0.38$ & $1.72 \pm 0.02$ \\
\hline 5.25 & $2.75 \pm 0.57$ & $2.76 \pm 0.57$ & $2.79 \pm 0.57$ & $2.78 \pm 0.57$ & $2.87 \pm 0.57$ & $2.79 \pm 0.05$ \\
\hline 7.75 & $3.84 \pm 0.82$ & $3.84 \pm 0.82$ & $3.85 \pm 0.82$ & $3.83 \pm 0.82$ & $3.84 \pm 0.82$ & $3.84 \pm 0.01$ \\
\hline 10.50 & $4.82 \pm 1.09$ & $4.84 \pm 1.09$ & $4.85 \pm 1.10$ & $4.85 \pm 1.10$ & $4.82 \pm 1.09$ & $4.84 \pm 0.02$ \\
\hline 13.00 & $6.04 \pm 1.36$ & $6.04 \pm 1.36$ & $6.07 \pm 1.37$ & $6.07 \pm 1.37$ & $6.05 \pm 1.37$ & $6.05 \pm 0.02$ \\
\hline 15.50 & $7.16 \pm 1.59$ & $7.16 \pm 1.61$ & $7.15 \pm 1.61$ & $7.15 \pm 1.62$ & $7.10 \pm 1.62$ & $7.14 \pm 0.03$ \\
\hline 21.00 & $8.88 \pm 2.01$ & $8.95 \pm 2.02$ & $8.95 \pm 2.03$ & $8.96 \pm 2.05$ & $8.96 \pm 2.06$ & $8.94 \pm 0.03$ \\
\hline 26.00 & $10.83 \pm 2.45$ & $10.94 \pm 2.44$ & $10.99 \pm 2.44$ & $11.03 \pm 2.49$ & $11.05 \pm 2.46$ & $10.97 \pm 0.09$ \\
\hline 31.50 & $12.79 \pm 3.06$ & $13.12 \pm 2.94$ & $13.14 \pm 2.89$ & $13.32 \pm 2.91$ & $12.29 \pm 2.55$ & $12.93 \pm 0.41$ \\
\hline
\end{tabular}

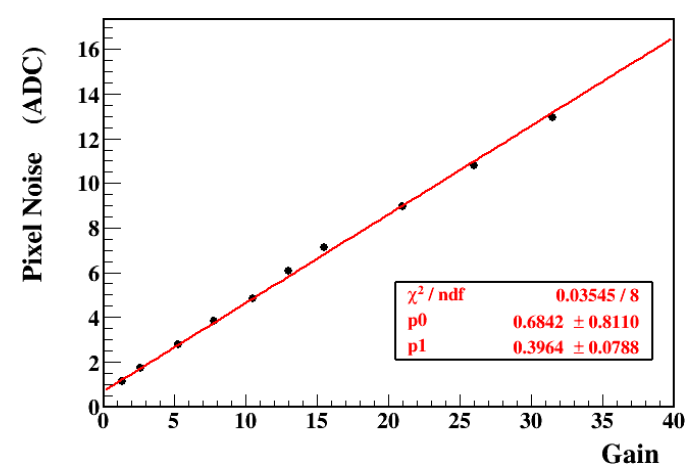

(a)

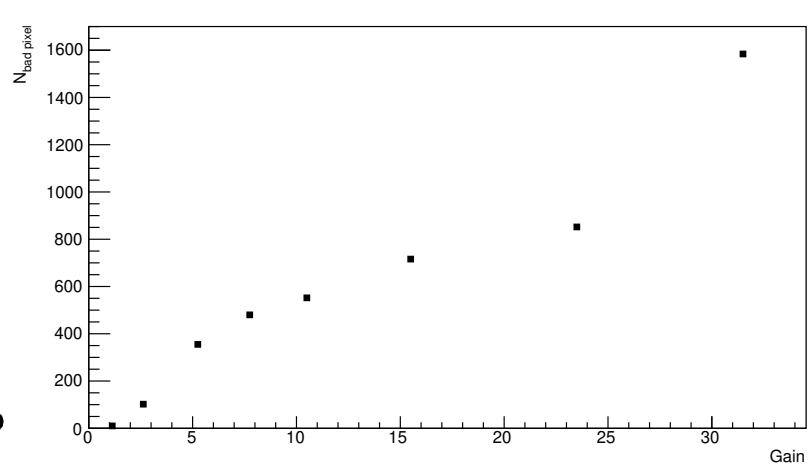

(b)

Figure 5. Mean noise (a) and number of bad pixels (b) as a function of Gain.

on the average single pixel noise nor on its RMS. Since all the variations are due to the change in gain, in the last column we reported the $\sigma$ averaged over all True Black values, whose RMS is at the level of $1 \%$. Figure 5a shows the dependence of the average pixel noise on the gain: found to be linear as expected. To ensure the maximum dynamic range for the signal, a low gain value has been chosen, in order to avoid the saturation of a single pixel by the signal given by an electron (see section 4.2 for more details).

Finally, the dependence on the gain value of the number of bad pixels ${ }^{1}$ has also been studied, and is shown in figure $5 \mathrm{~b}$. The increase of more than 3 orders of magnitude going from $G=1.31$ to $G=31.5$ is a clear indication of "problematic" channels that become visible at higher gain values.

${ }^{1}$ Pixels are defined bad when they fire a number of times that is not statistically compatible with the Poissonian expected fluctuations. They can be caused by many reasons: high leakage current, circuit defects, dust particles, scratches. Since their presence can alter the particle count, the algorithm identifies and removes them, as detailed in [20]. 
This is another indication for keeping the gain value as low as possible, hence the standard $G=1.31$ configuration with True Black $=42$ has been definitely chosen .

\subsubsection{Integration time}

As discussed above, the CMOS sensor is originally designed to be a camera, and thus it integrates the deposited energy over a time span $\left(\Delta T_{\text {frame }}\right)$ that can be varied. Increasing this time implies a higher number of particle tracks per each frame, and thus better statistics. At the same time, however, a longer $\Delta T_{\text {frame }}$ increases the single pixel noise level due to dark current. On the other hand, a too short $\Delta T_{\text {frame }}$ could bring to dead time issues due to the time needed by the data acquisition to write the event, with possible data losses.

To study this aspect, data were taken varying $\Delta T_{\text {frame }}$ in the $50-350 \mathrm{~ms}$ range with and without the presence of the ${ }^{90} \mathrm{Sr}$ source, acquiring average pedestal, noise and number of cluster. Results are shown in figure 6 .

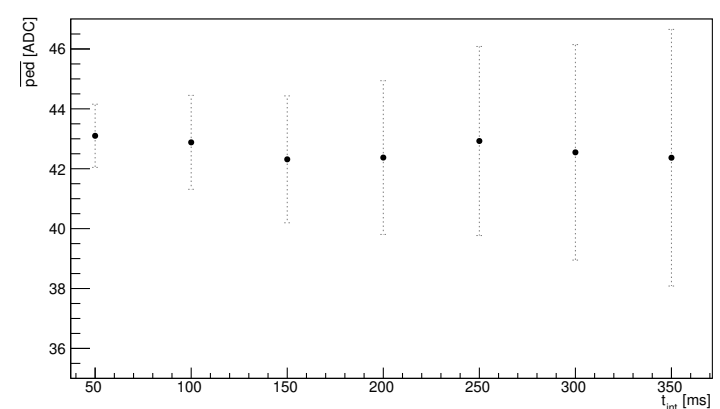

(a)

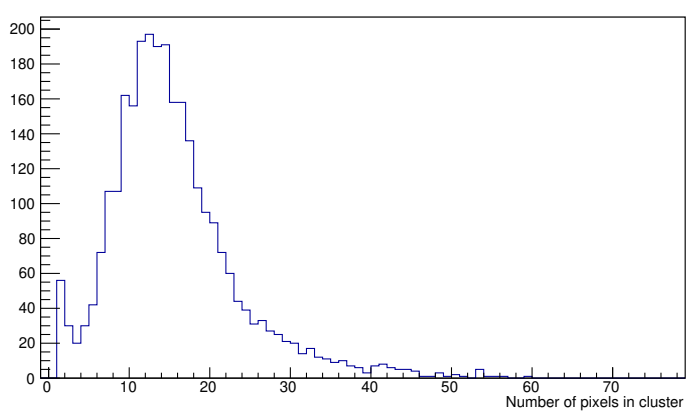

(c)

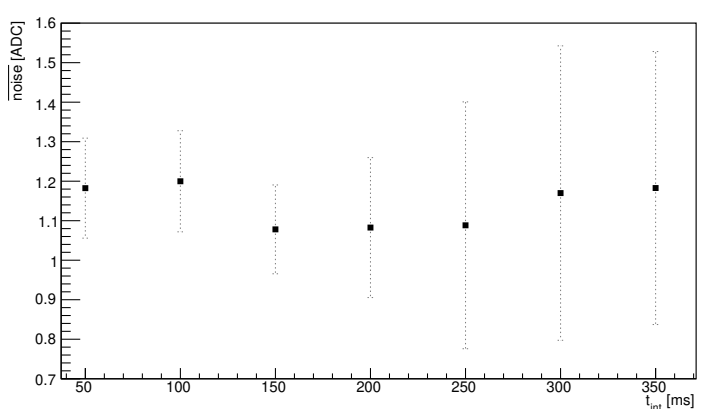

(b)

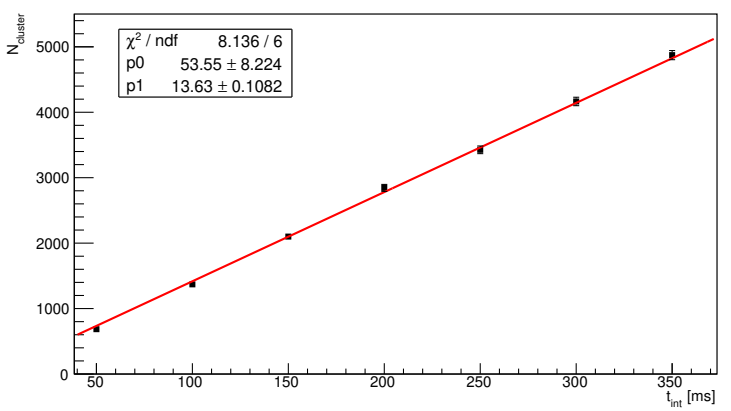

(d)

Figure 6. Test performed with ${ }^{90} \mathrm{Sr}$ source: mean pedestal (a), mean noise (b) variation as a function of integration time, with mean value error in solid black (hardly visible) and RMS in dashed gray. Number of pixels belonging to a cluster (c) for $G=1.31$ and number of cluster as a function of integration time (d).

As far as pedestals (figure 6a) and noise (figure 6b) are concerned, variations at $1 \%$ level are found, demonstrating their good stability. The clustering algorithm defined in [20] allows to group together pixels belonging to the same electron interaction with the sensor. The distribution of cluster width in terms of number of pixels is reported in figure $6 \mathrm{c}$, where the shape is the expected one. On the other hand, the number of signal clusters increases linearly with the integration time, as expected (figure 6d). To estimate the maximum systematic error, residuals of the linear fit were 
calculated, obtaining a value of $3.3 \%$. The choice of the working integration time is then not dictated by stability or efficiency issues, but mainly by the optimization of sensor operations. In fact, the interval should be long enough to increase the number of particles per frame, but also short compared to the surgeon movement timescale (few seconds), to allow several measurements of the flux in one position. We therefore confirmed the choice of $\Delta T_{\text {frame }}=200 \mathrm{~ms}$ already used previously.

\subsubsection{Temperature}

The detector should work in the operating room, which has a temperature of $\sim 22^{\circ}$. At the same time, it is expected to come also in contact with the patient, at around $36^{\circ}$. It is thus important that the response of the detector does not depend on the temperature in this range. To this aim, a scan with ${ }^{90} \mathrm{Sr}$ was performed in controlled environment with a temperature starting from $16^{\circ}$ and going up in $2-3^{\circ}$ steps to $39^{\circ}$, for a total of 11 acquisition.

Figure 7 shows results in terms of number of bad pixels (a), pedestal (b) and noise (c) mean values, as well as number of signal clusters (d) as a function of temperature. No relevant dependence on temperature has been found. In particular, the RMS of these distributions is $1.9 \%$, and can be assumed as the maximum systematic error: this is absolutely negligible in the considered application case.

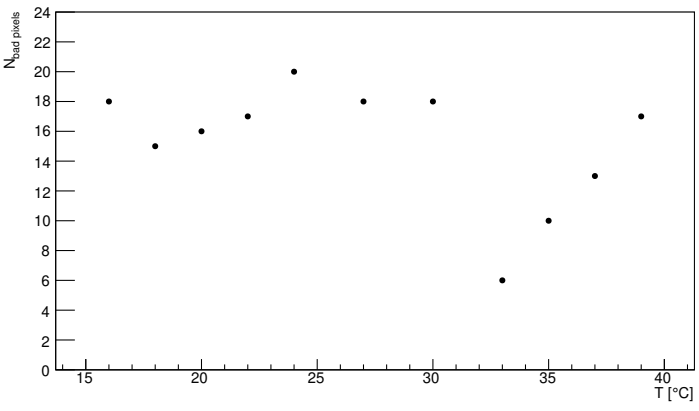

(a)

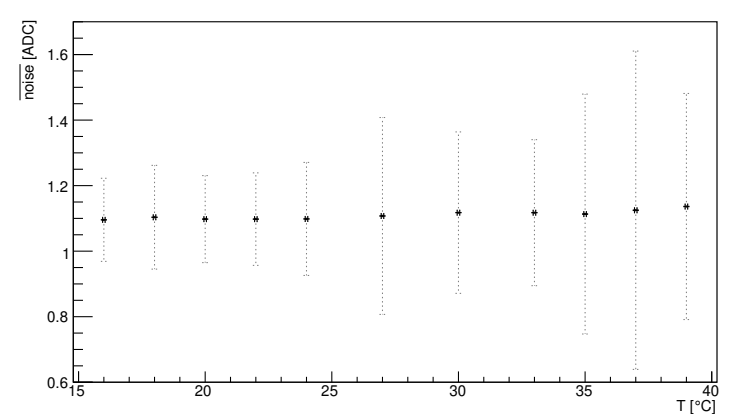

(c)

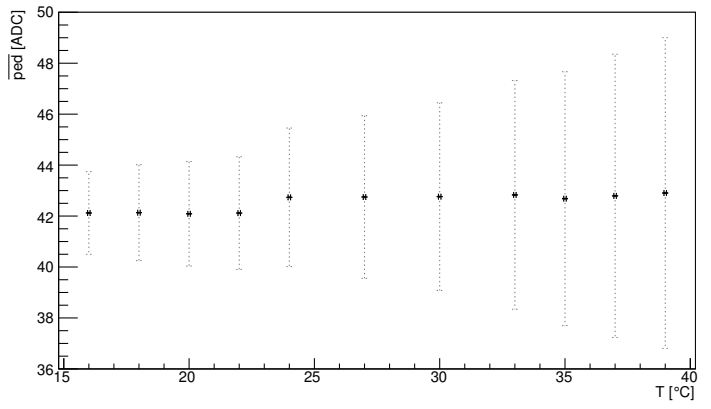

(b)

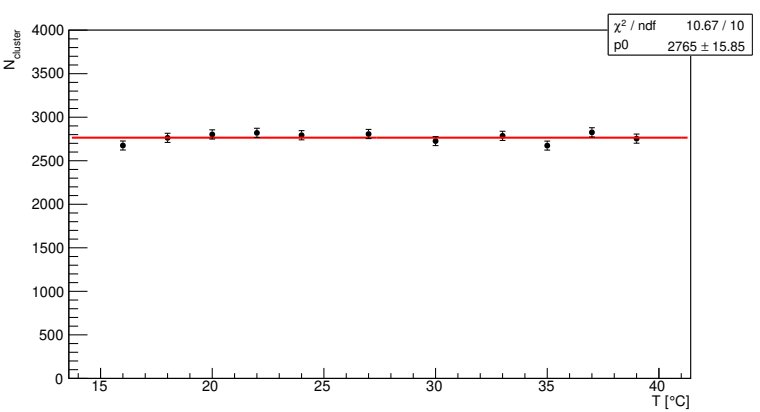

(d)

Figure 7. Number of bad pixels (a), mean pedestal (b), mean noise (c) and signal (d) variation as a function of temperature on ${ }^{90} \mathrm{Sr}$ source. For pedestal and noise both mean value error (in solid black) and RMS (in dashed gray) are reported, while for the number of cluster the error is the Poissonian one. 


\subsubsection{Time}

As it has been recently published in [23], the proposed $\beta^{-}$-RGS technique bases its efficacy on the definition of a "patient specific" counts threshold to discriminate what is probably tumor and what is not. Therefore, it is very important that the probe does not change its behaviour in time, considering that surgical procedures could in principle last several hours.

To investigate the stability, the setup has been placed inside a climate chamber set at $22^{\circ}$, and measurements have been taken for a long time period (22 days on ${ }^{90} \mathrm{Sr}$ source and 3 days on ${ }^{90} \mathrm{Y}$ source).

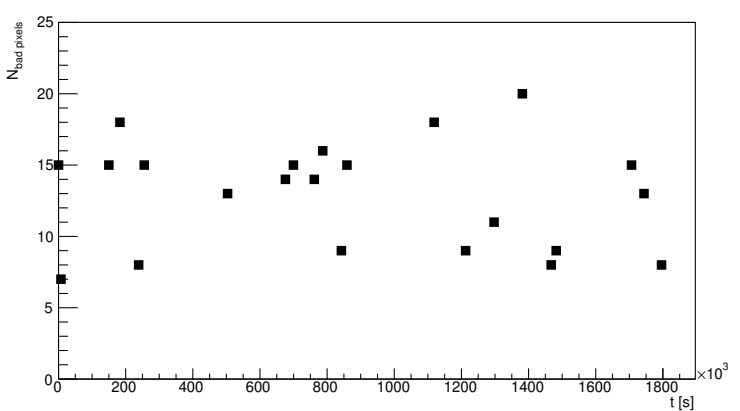

(a)

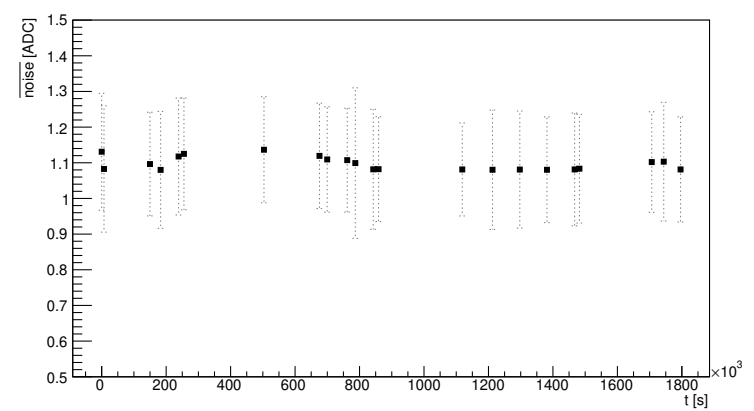

(c)

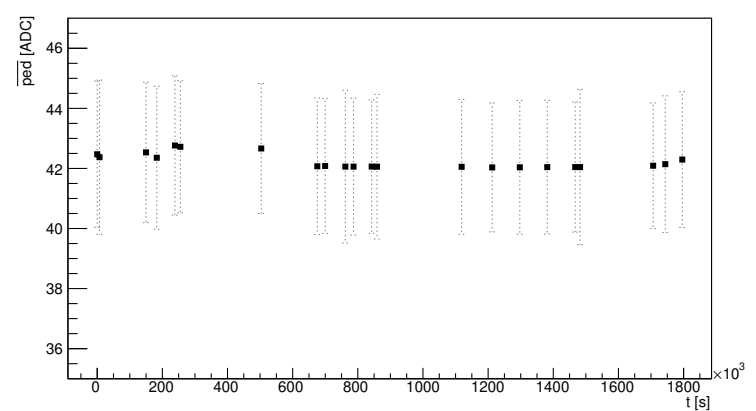

(b)

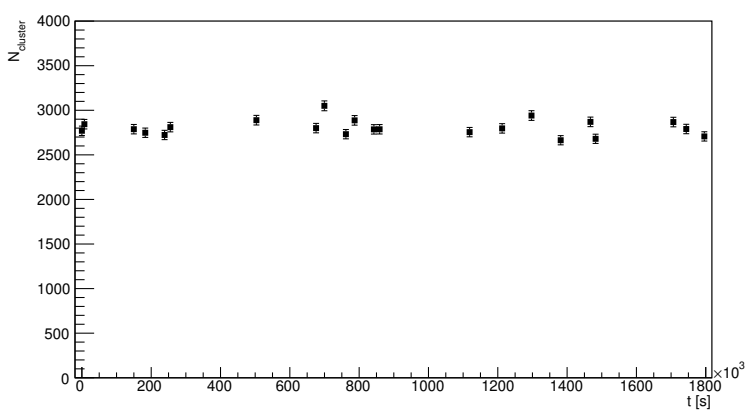

(d)

Figure 8. Number of bad pixels (a), mean pedestal (b), mean noise (c) and number of cluster (d) as a function of time on ${ }^{90} \mathrm{Sr}$ source. For pedestal and noise both mean value error (in solid black) and RMS (in dashed gray) are reported, while for the number of cluster the error is the Poissonian one.

Results for number of bad pixels (a), pedestal (b), noise (c) and number of signal cluster (d) in case of ${ }^{90} \mathrm{Sr}$ source are shown in figure 8 , and suggest that also in this case the variation is small, being evaluated again from a constant fit to be of the order of $3 \%$.

As far as the ${ }^{90} \mathrm{Y}$ source is concerned, the number of signal clusters is shown in figure 9. Points have been fitted with an exponential decay law $\left(A \cdot e^{-t / \tau}\right)$, obtaining $\tau=(94.6 \pm 2.9) \mathrm{h}$. Residuals with respect to expected $\tau_{\mathrm{Y}}=92 \mathrm{~h}$ allows to compute a systematic error of $2.2 \%$, which is again a tolerable error in view of the application context.

\subsection{Monte Carlo tuning}

The custom-made mechanical setup described above allows to easily interpose between the source and the detector small layers of absorbing materials. Several acquisitions were then taken by 


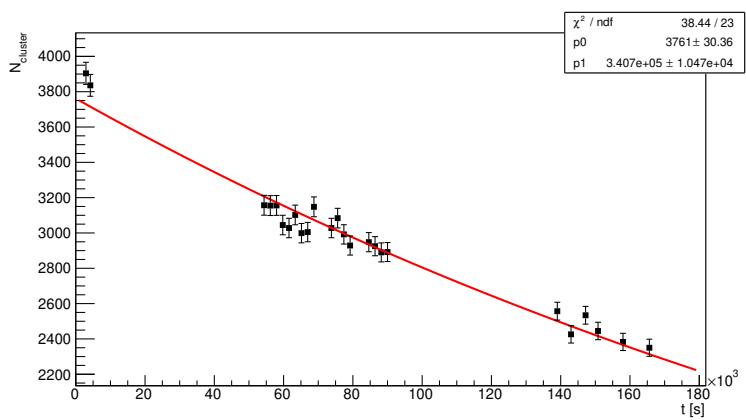

Figure 9. Number of signal cluster variation as a function of time on ${ }^{90} \mathrm{Y}$ source with Poissonian errors. The red line represents an exponential fit with $\tau=(94.6 \pm 2.9) \mathrm{h}$.

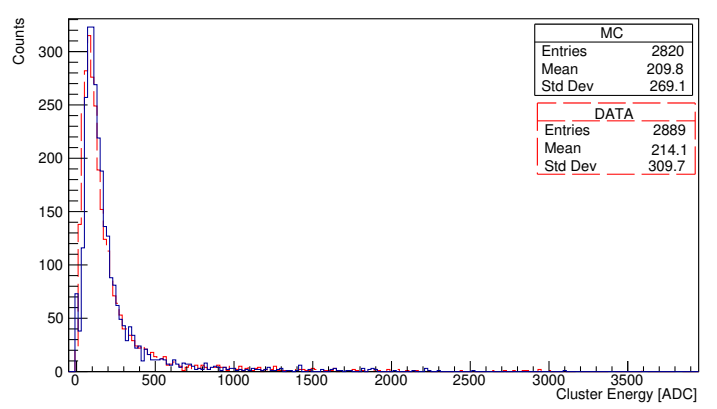

Figure 10. Tuning of the Monte Carlo simulation (solid blue) on the ${ }^{90} \mathrm{Sr}$ w/ absorber measurement (dashed red), taking the $3 \times 3$ cluster signal as comparison.

interposing $0.1 \mathrm{~mm}-0.2 \mathrm{~mm}-0.3 \mathrm{~mm}$ of Alluminum and $1.1 \mathrm{~mm}$ of ABS (Acrilonitrile Butadiene Stirene) between the ${ }^{90} \mathrm{Sr}$ source and the detector. The aforementioned $\gamma$ sources $\left({ }^{22} \mathrm{Na}\right.$ and $\left.{ }^{60} \mathrm{Co}\right)$ were also used. Such configurations are well reproducible and therefore can be used to validate the Monte Carlo (MC) simulations. As already discussed, the simulation's free parameters are the effective sensitive layer thickness $(\Delta Z)$ (no details on it being available in the sensor datasheet) and the conversion factor $(k)$ between deposited energy and ADC. The tuning of those parameters has been performed matching Monte Carlo results with experimental data in the ${ }^{90} \mathrm{Sr}-\mathrm{w} / \mathrm{o}$ absorber configuration. In particular, the distribution of the $3 \times 3$ cluster signal has been used as comparison (figure 10), and the best agreement both in terms of number of entries and shape has been found for $\Delta Z=1.75 \mu \mathrm{m}$ and $k=180 \mathrm{ADC} / \mathrm{keV}$.

Once tuned the Monte Carlo on the ${ }^{90} \mathrm{Sr}$ w/o absorber configuration, simulations have been performed also for the other absorbers and $\gamma$ sources, and results are shown, together with data, in figure 11. As a further comparison, keeping the same Monte Carlo tuning, a vertical scan has been performed on the ${ }^{90} \mathrm{Y}$ source, increasing the distance of the detector from the source from 2 to $5 \mathrm{~mm}$. Results are shown in figure 12, where Data and Monte Carlo are normalised to the first point to eliminate the significative uncertainty on the $\mathrm{Y}$ activity.

All results show a Data-MC agreement better than 5\%, and always within their respective uncertainties. Besides, it is worth highlighting that it is not so easy to reduce the uncertainty on simulation points, since this is not simply a "counting", which would scale with $\sqrt{N}, N$ being the number of simulated events, but is the result of the whole data analysis algorithm, that is ultimately 


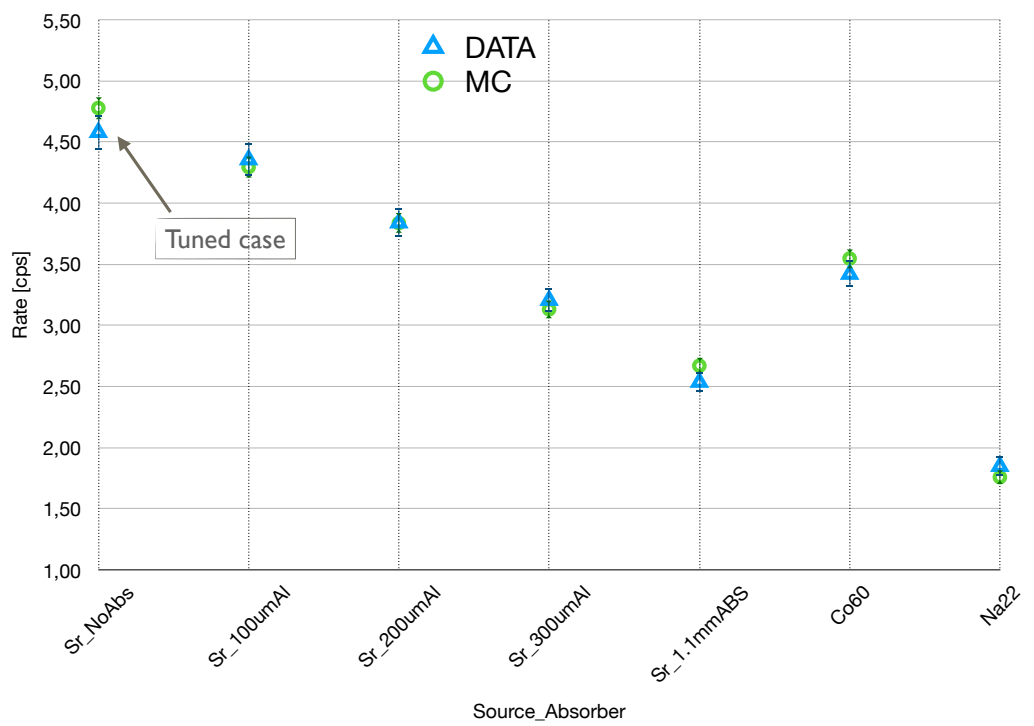

Figure 11. Number of clusters on several configurations of source and absorbers. Data (blue triangles) and MC (green circles) are shown, together with their Poissonian errors. Monte Carlo tuning has been performed only on the first configuration, as described in the text.

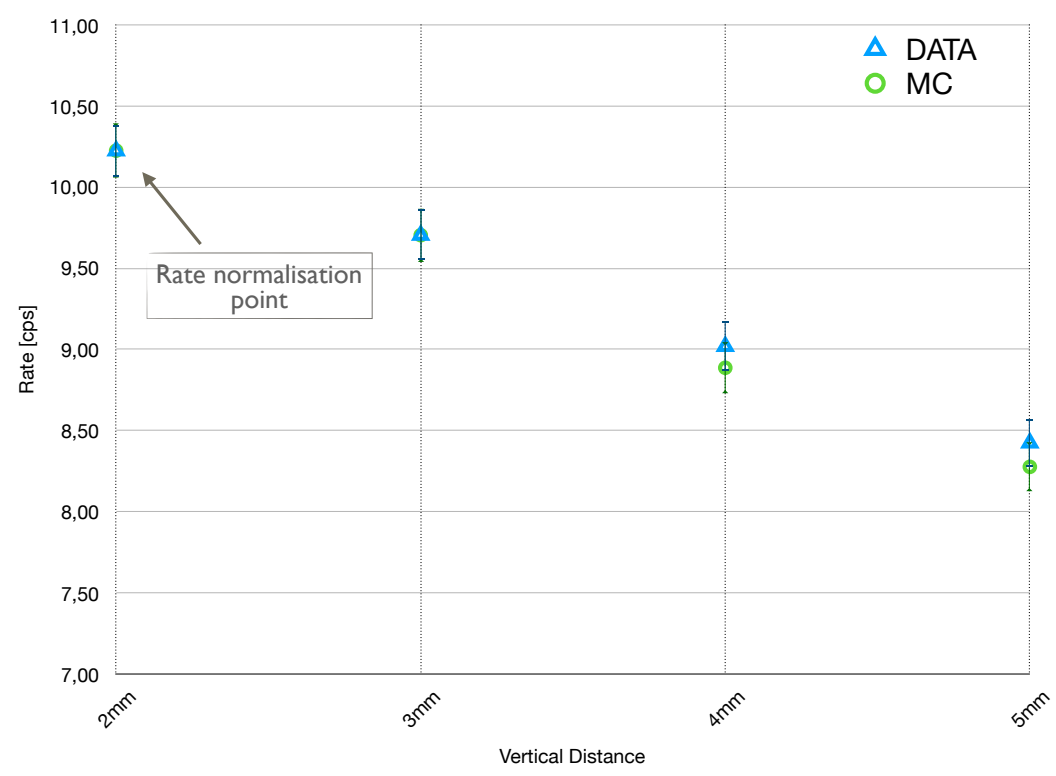

Figure 12. Number of clusters as a function of vertical distance from the ${ }^{90} \mathrm{Y}$ source. Data (blue triangles) and Monte Carlo (green circles) are normalised to the first point to eliminate the uncertainty on the Y absolute activity.

influenced by the topology of the energy release of the particle in the active area of the CIS (i.e. number of activated pixels).

\subsection{Efficiency curves}

The extremely good agreement found between data and Monte Carlo suggests that the developed simulation, once properly tuned, is an effective representation of the studied configuration. It is then 
possible to use it to asses the performances of the detector also in other experimental situations. In particular, to obtain efficiency curves on both electrons and photons, two different configurations have been simulated.

It has to be remembered that the expected typical application scenario for this detector is the one in which the probe is used to detect small tumor remnants, immersed in an almost isotropic background, constituted by photons coming from all the healthy organs nearby. These photons can in fact originate either from bremsstrahlung, in case of pure $\beta^{-}$emitters like ${ }^{90} \mathrm{Y}$, or from positron annihilation, in case of $\beta^{+}$emitters like ${ }^{18} \mathrm{~F}$ or ${ }^{68} \mathrm{Ga}$.

In this view, to evaluate the efficiency to beta particles, a Monte Carlo simulation has been performed with the detector placed in contact with a $16 \mathrm{~mm}$ diameter source layer of isotropic electrons having a flat energy distribution in the $0-2.3 \mathrm{MeV}$ range, to mimic the signal coming from the tumor ( $2.3 \mathrm{MeV}$ being the endpoint of ${ }^{90} \mathrm{Y}$ decay).

On the other hand, to evaluate the efficiency to background photons, a source of similar shape but emitting photons with a flat spectrum in the $0-1 \mathrm{MeV}$ range has been used.

To obtain the efficiency curves, the fraction of primary generated particles $\left(N_{\text {gen }}(E)\right)$ that originated an event that gave a "detected" signal (i.e. giving, after the discussed analysis algorithm, at least 1 cluster) in the active area of the detector $\left(N_{\text {det }}(E)\right)$ has been evaluated, for each energy bin.

In order to factorise out the geometrical efficiency $\left(\epsilon_{\text {geom }}\right)$, that is expected to vary from an application case to the other, the normalisation has been done with respect to particles having at least one "Monte Carlo step" into the active area of the detector $\left(N_{\mathrm{inCMOS}}(E)\right)$. In formula, for a given energy bin (E):

$$
\epsilon_{\mathrm{tot}}(E)=\frac{N_{\mathrm{det}}(E)}{N_{\mathrm{gen}}(E)}=\frac{N_{\mathrm{det}}(E)}{N_{\mathrm{inCMOS}}(E)} \cdot \frac{N_{\mathrm{inCMOS}}(E)}{N_{\mathrm{gen}}(E)}=\epsilon_{\mathrm{det}}(E) \cdot \epsilon_{\mathrm{geom}} .
$$

The aim of this study is then to evaluate the detection efficiency as a function of the primary particle energy:

$$
\epsilon_{\text {det }}(E)=\frac{N_{\text {det }}(E)}{N_{\text {inCMOS }}(E)} .
$$

It has however to be remembered that the CMOS sensor used in this study is shielded by a $400 \mu \mathrm{m}$ layer of $\rho=2.4 \mathrm{~g} / \mathrm{cm}^{3}$ density glass for protection purposes, that was not possible to remove before measurements. Nevertheless, in the Monte Carlo it is possible to study the behaviour of the detector with a thinner protective layer, or even without it. The way to obtain the efficiency curve, however, does depend on whether the protective layer is present or not, due to the subsequent change in geometry.

\subsubsection{Without protective layer}

When there is no protective layer, the source and the detector are put in contact (figure 13, top). The denominator of eq. (4.2) is given by the energy spectrum of primary particles that perform at least one "MC step" in the active volume (directly or via secondaries). The numerator, on the other hand, is given by the energy spectrum of primary particles that, directly or again via their secondaries, give a detected (i.e. above threshold) signal.

It has to be highlighted that while the former distribution is directly obtained from the simulation, to obtain the latter all the analysis algorithm, previously described in the text, must be applied, 

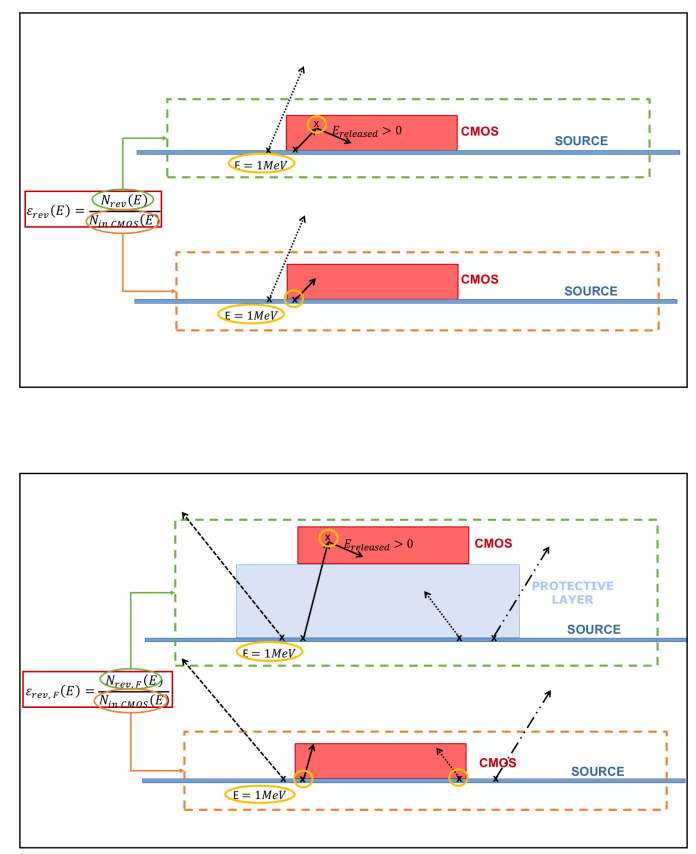

Figure 13. Scheme (not to scale) of the configurations simulated to obtain efficiency curves, for a detector w/o (above) and w/ (bottom) protective layer, as described in text.

including random noise generation and subtraction and so on. The key aspect in this case is that the information of the energy of the primary particle that originated the one that is giving a detected signal is carried up to the end of the analysis algorithm. In this way, there is also no risk to suffer from double counting, if for example a $2 \mathrm{MeV}$ primary electron originates two different particles which are both detected, in which case only one entry in the efficiency curve would be added.

To finally compute the efficiency, the two histograms are divided bin-by-bin, and results are shown in figure 14 and 15.

As expected, once the protective layer is removed, the CMOS has very high efficiency even for low energy electrons, reaching $95 \%$ at $\sim 100 \mathrm{keV}$. At the same time, the sensitivity to photons remains low, at about $4 \%$, that is about one order of magnitude smaller than what is achieved by the original scintillator-based detector [8].

\subsubsection{With protective layer}

When the detector is simulated together with its actual protective layer, the definition of $N_{\text {det }}(E)$ needs to be modified. In this case, in fact, the same extended flat-energy source is placed in contact with the protective layer (figure 13, bottom). As far as the denominator is concerned, since we want to normalise the amount of particles that give a detected signal to the number of those that could actually reach the detector, the same simulation of the "without protective layer" case was used. In this case, the assumption is that the change in solid angle due to the source-CMOS distance given by the presence of the protective layer can be neglected, due to the traverse dimensions of the source, that are much greater than the change in distance. Results both for electrons and photons are shown in figure 16. Again, as expected, when the heavy protective layer is present in front of the CMOS, 

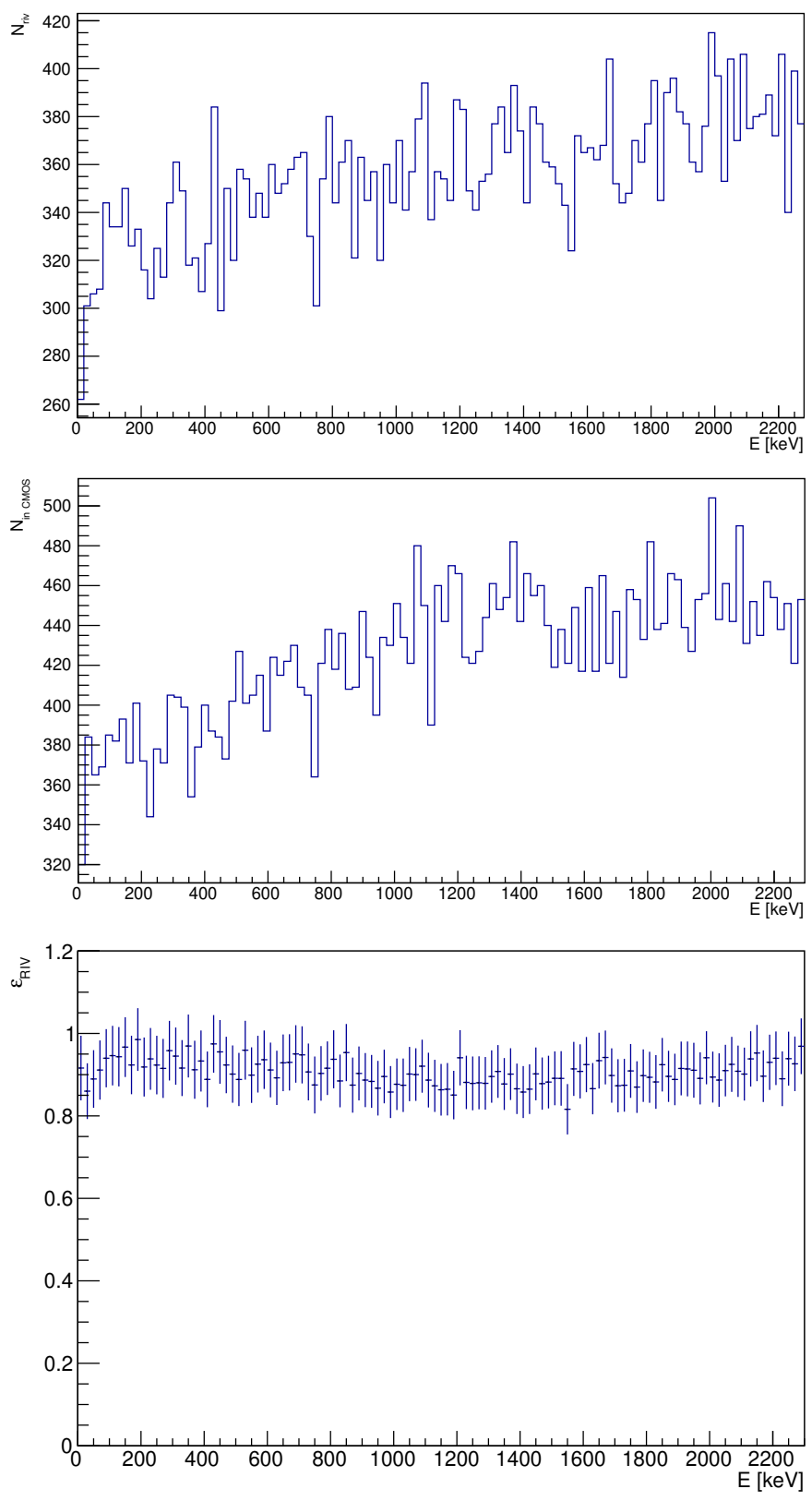

Figure 14. Numerator (top) and denominator (middle) of the fraction expressing the efficiency (see eq. (4.2)), as described in the text, and final efficiency for electrons (bottom), in the case w/o protective layer.

the detector is substantially blind to electrons with energy smaller than $400 \mathrm{keV}$, reaching $80 \%$ sensitivity only at $900 \mathrm{keV}$. In the same time, the protective layer does also act like a "converter" for photons, that in fact have a higher probability to be converted into electrons in the protective layer and be thus detected by the active area with respect to the $\mathrm{w} /$ protective layer case, reaching a plateau of about $1 \%$.

Finally, to quantify the effect of a possible shrinking of the protective layer thickness, several Monte Carlo simulations have been performed variating it. Results for electron efficiency are shown in figure 17, and clarify the remarkable effect of even small layers of absorbers in front of the active 

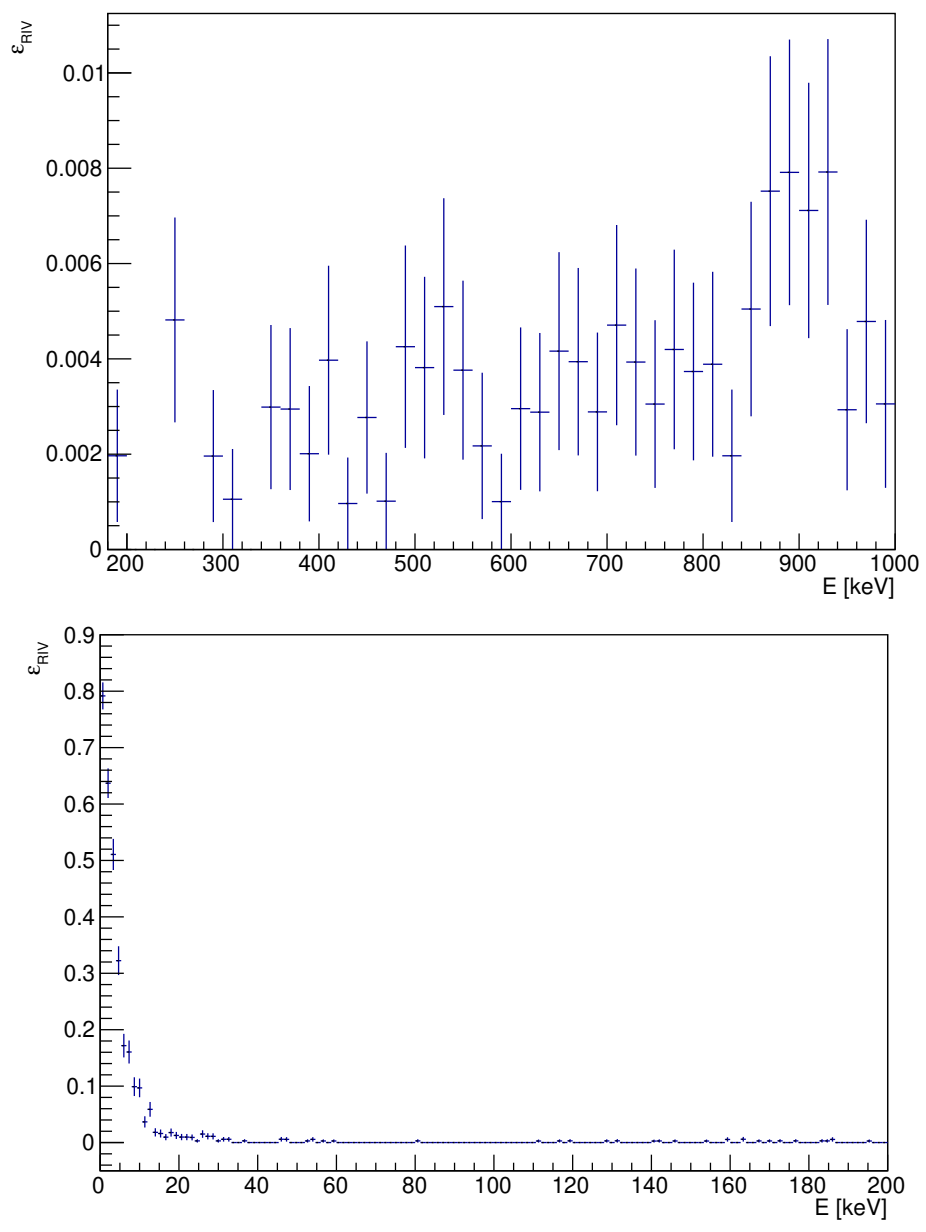

Figure 15. Final efficiency for photons, in the case w/o protective layer. The lower plot is a zoom of the low energy region.

area of the detector. This aspect has also te be carefully considered in view of the design of the final probe casing, were additional layers of materials are expected in front of the CIS.

\subsection{Conclusions}

A novel Radio Guided Surgery technique has been recently proposed, based on the detection of low energy electrons. For such a technique to be developed, a detector is needed to reveal during surgery electrons emitted by tumors, being transparent to bremsstrahlung photons that constitute a background. First tests have been carried out with a scintillator-based probe, which proved to have an efficiency to electrons reaching $70 \%$ at about $540 \mathrm{keV}$, being substantially blind below $\sim 300 \mathrm{keV}$. The use of CMOS Image Sensor as possible active area of the detector has thus been proposed, with the aim to improve the sensitivity also to low energy electrons.

In this study, we characterised a commercial sensor (MT9V115 from MICRON (now Aptina Imaging), $648 \times 488 \mathrm{px}$, pixel size $1.75 \mu \mathrm{m}$ ) in terms of stability over time, temperature, gain, and integration time. By means of several measurements on radioactive sources, a dedicated Monte Carlo Geant 4 simulation has been validated, and efficiency to $\beta$ and $\gamma$ particles evaluated. 

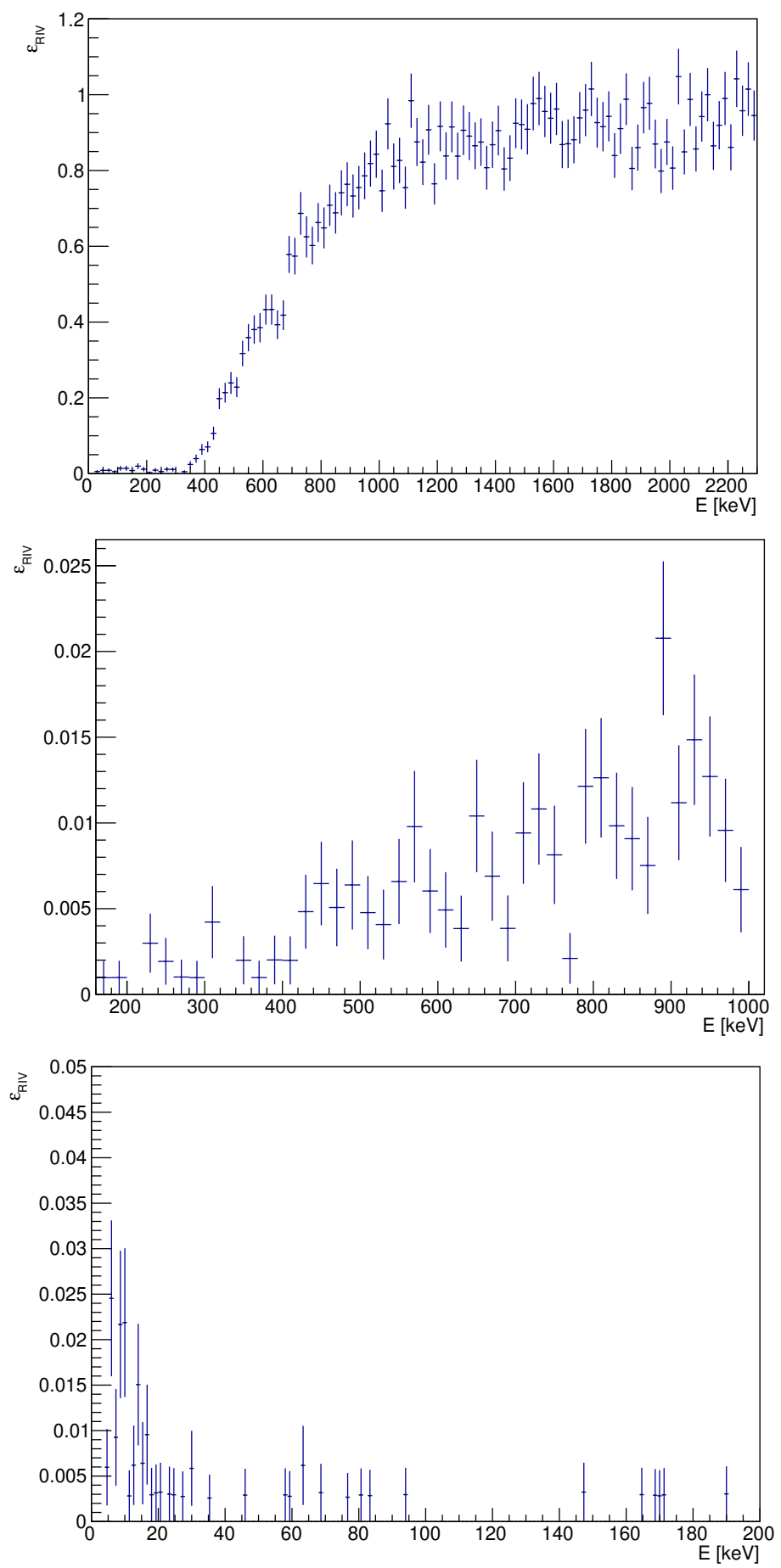

Figure 16. Efficiency for electrons (top) and photons in the case w/ protective layer (middle). Bottom plot shows a low energy zoom for photons efficiency.

The sensor proved to be stable enough in the needed operational range of time and temperature, and the optimal Gain and integration time have been identified. As far as the efficiency are concerned, the "off the shelf" sensor is not a good candidate. In fact, it comes with a $400 \mu \mathrm{m}$ thick layer of $2.4 \mathrm{~g} / \mathrm{cm}^{3}$ density glass in front, that acts as absorber for exactly those low energy particles 


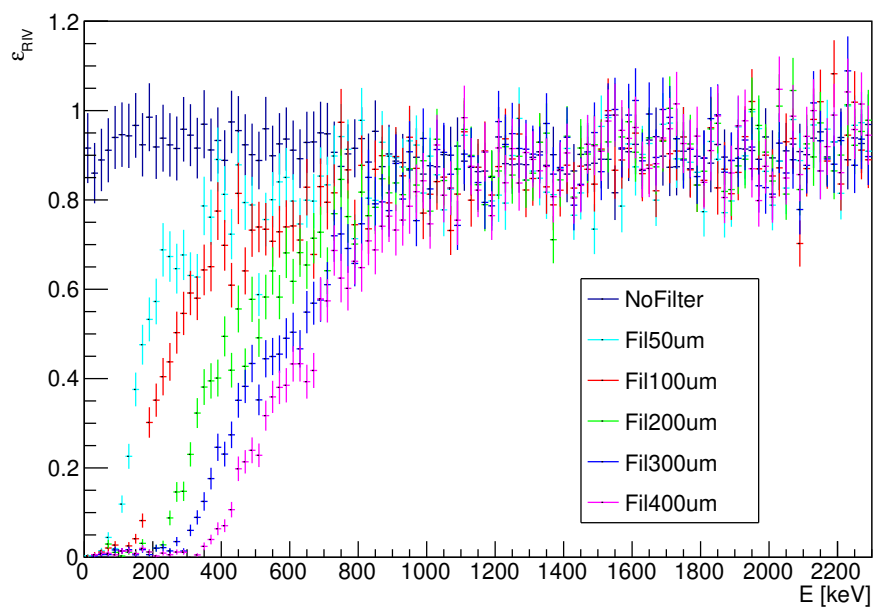

Figure 17. Electron efficiency varying the thickness of the protective layer, highlighting the ameliorating of its reduction.

that are here tailored. Moreover, the protective layer does also convert photons, that would have passed through the detector, into electrons, much more easily revealed, resulting in an increased sensitivity to the background.

However, thanks to the Monte Carlo simulation that has been developed and validated, finding very good agreement with respect to experimental data, it is possible to study the efficiency of a possible detector to which the protective layer has been removed. In such configuration the efficiency to electrons is very high $(>90 \%)$ also at low energy $(\sim 100 \mathrm{keV})$ electrons. At the same time the sensitivity to photons is of the order of few $\%$, about one order of magnitude smaller than the one of the scintillating-based detector.

In conclusion, a CMOS based detector for Radio Guided Surgery could be incredibly effective in revealing low energy electrons, thus allowing the technique to be performed with a wider variety of radio tracers, therefore enlarging the amount of possible application cases. Moreover, a higher sensitivity detector could allow also to reduce the amount of activity to inject to the patient, with all the consequences in terms of absorbed dose to him/her and to the medical personnel.

To this goal, however, commercially available sensor are not a viable option, due to the presence of a protective layer. For the next future, tests are foreseen to confirm the effect of thinning of this layer, suggested by Monte Carlo simulations.

\section{References}

[1] E. Solfaroli Camillocci et al., A novel radioguided surgery technique exploiting $\beta^{-}$decays, Sci. Rep. 4 (2014) 4401 [arXiv: 1402.2248].

[2] S.P. Povoski et al., A comprehensive overview of radioguided surgery using gamma detection probe technology, World J. Surg. Oncol. 7 (2009) 11.

[3] M. Tsuchimochi and K. Hayama, Intraoperative gamma cameras for radioguided surgery: Technical characteristics, performance parameters, and clinical applications, Phys. Med. 29 (2013) 126. 
[4] E. Solfaroli Camillocci et al., An Intraoperative $\beta$-Detecting Probe For Radio-Guided Surgery in Tumour Resection, IEEE Trans. Nucl. Sci. 63 (2016) 2533 [arXiv: 1511. 02059].

[5] E. Solfaroli Camillocci et al., First ex-vivo validation of a radioguided surgery technique with $\beta$-radiation, Phys. Med. 32 (2016) 1139.

[6] A. Russomando et al., The $\beta$-radio-guided surgery: Method to estimate the minimum injectable activity from ex-vivo test, Phys. Med. 58 (2019) 114.

[7] F. Collamati et al., Radioguided surgery with $\beta$-radiation in pancreatic Neuroendocrine Tumors: a feasibility study, Sci. Rep. 10 (2020) 1.

[8] C. Mancini-Terracciano et al., Feasibility of beta-particle radioguided surgery for a variety of "nuclear medicine" radionuclides, Phys. Med. 43 (2017) 127.

[9] M. Angelone et al., Properties of para-Terphenyl as Detector for $\alpha, \beta$ and $\gamma$ radiation, IEEE Trans. Nucl. Sci. 61 (2014) 1483 [arXiv: 1305.0442].

[10] M. El Lakis et al., Radioguided Surgery With Gallium 68 Dotatate for Patients With Neuroendocrine Tumors, JAMA Surg. 154 (2019) 40.

[11] F. Collamati et al., Characterisation of a $\beta$ detector on positron emitters for medical applications, Phys. Med. 67 (2019) 85.

[12] F. Collamati et al., Radioguided surgery with $\beta$ radiation: a novel application with $\mathrm{Ga}^{68}$, Sci. Rep. 8 (2018) 16171.

[13] L. Servoli, D. Biagetti, D. Passeri and E.S. Gattuso, Use of Standard CMOS Pixel Imagers as Ionizing Radiation Detectors, in proceedings of the 2008 IEEE Nuclear Science Symposium Conference Record, Dresden, Germany, 19-25 Octobe 2008, pp. 2484-2488.

[14] M. Esposito, T. Anaxagoras, O. Diaz, K. Wells and N.M. Allinson, Radiation hardness of a large area CMOS active pixel sensor for bio-medical applications, in proceedings of the 2012 IEEE Nuclear Science Symposium and Medical Imaging Conference Record (NSS/MIC), Anaheim, CA, U.S.A., 27 October-3 November 2012, pp. 1300-1304.

[15] E. Conti et al., Use of a CMOS Image Sensor for an Active Personal Dosimeter in Interventional Radiology, IEEE Trans. Instrum. Meas. 62 (2013) 1065.

[16] S. Meroli, D. Passeri and L. Servoli, Energy loss measurement for charged particles in very thin silicon layers, 2011 JINST 6 P06013.

[17] L. Servoli, D. Biagetti, S. Meroli, P. Placidi, D. Passeri and P. Tucceri, Use of a standard CMOS imager as position detector for charged particles, Nucl. Phys. B Proc. Suppl. 215 (2011) 228.

[18] https://www.onsemi.com.

[19] R. Brun and F. Rademakers, ROOT: An object oriented data analysis framework, Nucl. Instrum. Meth. A 389 (1997) 81.

[20] L.A. Solestizi et al., Use of a CMOS image sensor for beta-emitting radionuclide measurements, 2018 JINST 13 P07003.

[21] L. Servoli, D. Biagetti, S. Meroli, P. Placidi, D. Passeri and P. Tucceri, Use of a standard CMOS imager as position detector for charged particles, Nucl. Phys. B Proc. Suppl. 215 (2011) 228.

[22] J. Allison et al., Recent developments in Geant4, Nucl. Instrum. Meth. A 835 (2016) 186.

[23] S. Morganti et al., Tumor-non-tumor discrimination by a $\beta$-detector for Radio Guided Surgery on ex-vivo neuroendocrine tumors samples, Phys. Med. 72 (2020) 96. 